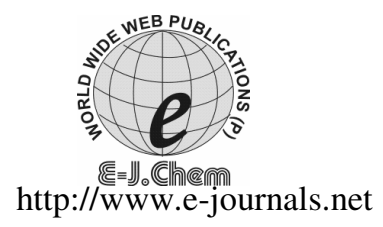

ISSN: 0973-4945; CODEN ECJHAO

E-Journal of Chemistry

2009, 6(S1), S473-S479

\title{
Synthesis and Antimicrobial Activity of Amino Acids Conjugated Diphenylmethylpiperazine Derivatives
}

\author{
K N. SHIVAKUMARA, K C. PRAKASHA and D. CHANNE GOWDA* \\ Department of Studies in Chemistry, \\ University of Mysore, Manasagangotri, Mysore-570006, India. \\ dchannegowda@yahoo.co.in
}

Received 6 May 2009; Accepted 1 July 2009

\begin{abstract}
A series of amino acid conjugated diphenylmethylpiperazine derivatives were synthesized by coupling diphenylmethylpiperazine with different Boc-amino acids using EDCI/HOBt as coupling agent and NMM as base. The synthesized compounds were characterized by ${ }^{1} \mathrm{H}-\mathrm{NMR}$ and elemental analysis. The Boc-deblocked derivatives were tested for their antimicrobial activity. We are here reporting that Phe and Trp conjugated diphenylmethylpiperazine showed equally good antibacterial activities as that of conventional antimicrobial drugs.
\end{abstract}

Keywords: Antimicrobial drugs, Diphenylmethylpiperazine (Benzhydrilpiperazine), Conjugated amino acids and Synthesis.

\section{Introduction}

Piperazine and their derivatives have their own importance in today's drug discovery. Piperazine moiety certainly deserves the molecule backbone with versatile binding properties representing potent and selective ligands for a range of different biological targets in medicinal chemistry. Thus, piperazine is considered as honored scaffold. A number of substituted piperazines posses significant pharmacological action such as antihistamic ${ }^{1-2}$ antimicrobial, ${ }^{3}$ acetylcholinesterae inhibitors ${ }^{4}$, antimalarial ${ }^{5}$, dopamine transporter ${ }^{6-7}, \mathrm{D}_{2} / \mathrm{D}_{4}$ antagonist ${ }^{8}, \quad \mathrm{MC}_{4}$ Receptor $^{9}$, and $\mathrm{HIV}$-protease inhibitor ${ }^{10-11}$. Under this category, benzhydrilpiperazine (diphenylmethylpiperazine) belongs to the diarylpiperazine family; possesses wide range of pharmacological properties such as anti-lipid-peroxidation activity $^{12}$, antiallergic and antioxidant activity ${ }^{13}$ antihistamic activity ${ }^{14}$, myocardiuminhibiting agent ${ }^{15}$ and antimicrobial activity ${ }^{16-17}$.

Currently there is a tendency to use amino acid/peptidyl residues during the prodrug design process. The literature reports that bioactive compounds show enhanced activity when linked to amino acids ${ }^{18-23}$. The presence of an unusual amino acid and heterocyclic 
building blocks has stimulated interest in new synthetic methodologies and strategies to obtain a target structure. In this connection, we have synthesized novel diphenylmethylpiperazine derivatives by the coupling of diphenylmethylpiperazine with various $\mathrm{N}$-protected amino acids. The present study was undertaken with a view to find the efficacy of these diphenylmethylpiperazine derivatives as antimicrobial agents.

\section{Materials and methods}

All the amino acids used were of $L$-configuration unless mentioned. All Boc-amino acids and HOBt were purchased from Advanced Chem. Tech. (Louisville, Kentuky, USA). EDCI and NMM were purchased from Sigma Chemicals Co. (St. Louis, MO). All solvents and reagents used for the synthesis and analysis were of analytical grade. TLC was carried out on precoated silica gel plates (Merck) using chloroform/methanol/acetic acid (95:5:3) as an eluting system. ${ }^{1} \mathrm{H}$ NMR spectra were obtained on a $300 \mathrm{MHz}$ Bruker FT-NMR Spectrometer instrument by using $\mathrm{CDCl}_{3}$ as solvent and TMS as an internal standard.

The diphenylmethylpiperazine was synthesized as previously reported using standard procedure ${ }^{24}$. The compound 4 was coupled with different Boc-amino acids using EDCI/HOBt as coupling agent and NMM as base (Scheme 1).

\section{Synthesis}

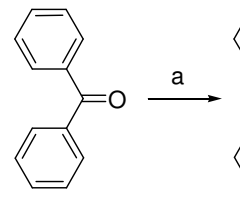

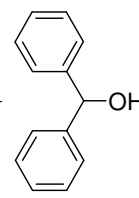

2

b

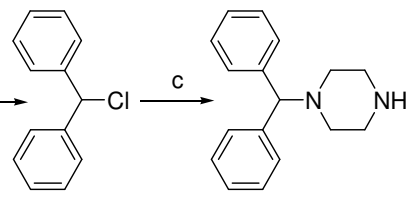

3

Where, $\mathrm{R}=$ Side chains of amiono acids

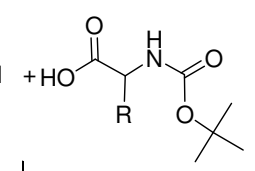

d

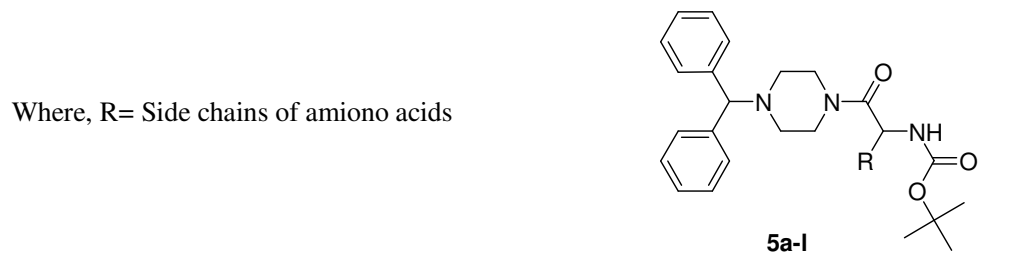

Scheme 1. Reagents and conditions (a) $\mathrm{NaBH}_{4}$, methanol, r.t, $5 \mathrm{~h}$ (b) $\mathrm{SOCl}_{2}$, dichloromethane, $0-5{ }^{0} \mathrm{C}, 4 \mathrm{~h}$ (c) piperazine, $\mathrm{K}_{2} \mathrm{CO}_{3}$, DMF, $80{ }^{\circ} \mathrm{C}, 8 \mathrm{~h}$. (d) EDCl, HOBt, NMM, DMF, $0{ }^{\circ} \mathrm{C}, 24 \mathrm{~h}$.

General procedure for the coupling of N-Boc amino acids with diphenylmethylpiperazine

To the stirred solution of Boc-amino acid $(2 \mathrm{mmol})$ and HOBt $(0.31 \mathrm{~g}, 2 \mathrm{mmol})$ in DMF $(10 \mathrm{~mL})$ cooled to $0{ }^{\circ} \mathrm{C}$, added NMM $(0.22 \mathrm{~mL}, 2 \mathrm{mmol})$, EDCI $(0.42 \mathrm{~g}, 2 \mathrm{mmol})$ and diphenylmethylpiperazine $(0.5 \mathrm{~g}, 2 \mathrm{mmol})$. After 20 minutes, the $\mathrm{pH}$ of solution was adjusted to 8 by the addition of NMM and the reaction mixture was stirred overnight while slowly warming to room temperature. The reaction mixture was quenched with water $(2 \mathrm{~mL})$ and the solvent was condensed. The residue was dissolved in chloroform $(25 \mathrm{~mL})$, washed with $5 \% \mathrm{NaHCO}_{3}$ $(3 \times 20 \mathrm{~mL}), \mathrm{H}_{2} \mathrm{O}(1 \times 20 \mathrm{~mL})$ followed by $0.1 \mathrm{~N}$ cold $\mathrm{HCl}(3 \times 20 \mathrm{~mL})$ and brine solution $(3 \times$ $20 \mathrm{~mL}$ ), dried over anhydrous $\mathrm{Na}_{2} \mathrm{SO}_{4}$. The chloroform was removed under reduced pressure to obtain the desired products. The analytical data of these compounds are presented in (Table 1). 
Table 1. Analytical data of the synthesized compounds.

\begin{tabular}{|c|c|c|c|c|c|c|c|}
\hline \multirow{2}{*}{ Entry } & \multirow{2}{*}{$\begin{array}{l}\text { Side chain of amino } \\
\text { acids (R) }\end{array}$} & \multirow{2}{*}{$\begin{array}{c}\text { Yield } \\
\%\end{array}$} & \multirow{2}{*}{$\begin{array}{l}\text { Molecular } \\
\text { formula }\end{array}$} & \multicolumn{3}{|c|}{ Elemental analysis, $\%$} & \multirow{2}{*}{${ }^{1} \mathrm{H}-\mathrm{NMR}\left(\mathrm{CDCl}_{3}\right), \delta$} \\
\hline & & & & $\mathrm{C}$ & $\mathrm{H}$ & $\mathrm{N}$ & \\
\hline $5 a$ & & 90 & $\mathrm{C}_{24} \mathrm{H}_{31} \mathrm{~N}_{3} \mathrm{O}_{3}$ & $\begin{array}{c}70.38 \\
(70.41)\end{array}$ & $\begin{array}{c}7.55 \\
(7.57)\end{array}$ & $\begin{array}{c}10.24 \\
(10.26)\end{array}$ & $\begin{array}{l}\text { 7.05-7.25 (m, 10H, Ar-H); } 1.39(\mathrm{~s}, 9 \mathrm{H}, \mathrm{Boc}) ; 7.91(\mathrm{~s}, 1 \mathrm{H}, \\
\mathrm{NH}-\mathrm{Boc}) ; 4.62(\mathrm{~s}, 1 \mathrm{H},-\mathrm{CH}-) ; 2.84\left(\mathrm{~s}, 4 \mathrm{H},-\mathrm{CH}_{2^{-}}\right) ; 2.60(\mathrm{~s}, 4 \mathrm{H}, \\
\left.-\mathrm{CH}_{2^{-}}\right) ; 1.93\left(\mathrm{~s}, 2 \mathrm{H}, \alpha \mathrm{CH}_{2}\right) .\end{array}$ \\
\hline $5 b$ & & 95 & $\mathrm{C}_{25} \mathrm{H}_{33} \mathrm{~N}_{3} \mathrm{O}_{3}$ & $\begin{array}{c}70.91 \\
(70.92)\end{array}$ & $\begin{array}{c}7.88 \\
(7.80)\end{array}$ & $\begin{array}{c}9.92 \\
(9.92)\end{array}$ & $\begin{array}{l}\text { 7.05-7.25 (m, 10H, Ar-H); } 1.39(\mathrm{~s}, 9 \mathrm{H}, \mathrm{Boc}) ; 7.93(\mathrm{~s}, 1 \mathrm{H}, \mathrm{NH}- \\
\mathrm{Boc}) ; 4.62(\mathrm{~s}, 1 \mathrm{H},-\mathrm{CH}-) ; 2.88\left(\mathrm{~s}, 4 \mathrm{H},-\mathrm{CH}_{2^{-}}\right) ; 2.62(\mathrm{~s}, 4 \mathrm{H},- \\
\left.\mathrm{CH}_{2^{-}}\right) ; 4.33(\mathrm{~s}, 1 \mathrm{H}, \alpha \mathrm{CH}) ; 1.25\left(\mathrm{~d}, 3 \mathrm{H}, \beta_{\left.\mathrm{CH}_{3}\right)}\right)\end{array}$ \\
\hline $5 c$ & & 93 & $\mathrm{C}_{27} \mathrm{H}_{37} \mathrm{~N}_{3} \mathrm{O}_{3}$ & $\begin{array}{l}71.83 \\
(71.84)\end{array}$ & $\begin{array}{c}8.22 \\
(8.20)\end{array}$ & $\begin{array}{c}9.30 \\
(9.31)\end{array}$ & $\begin{array}{l}\text { 7.05-7.27 (m, 10H, Ar-H); } 1.39(\mathrm{~s}, 9 \mathrm{H}, \mathrm{Boc}) ; 7.90(\mathrm{~s}, 1 \mathrm{H}, \mathrm{NH}- \\
\mathrm{Boc}) ; 4.65(\mathrm{~s}, 1 \mathrm{H},-\mathrm{CH}) ; 2.84\left(\mathrm{~s}, 4 \mathrm{H},-\mathrm{CH}_{2^{-}}\right) ; 2.60(\mathrm{~s}, 4 \mathrm{H},- \\
\left.\mathrm{CH}_{2^{-}}\right) ; 4.51(\mathrm{~d}, 1 \mathrm{H}, \alpha \mathrm{CH}-) ; 1.71(\mathrm{~m}, 1 \mathrm{H}, \beta \mathrm{CH}-) ; 1.25(\mathrm{~d}, 6 \mathrm{H}, \\
\left.\gamma \mathrm{CH}_{3}\right) .\end{array}$ \\
\hline $5 \mathrm{~d}$ & & 91 & $\mathrm{C}_{28} \mathrm{H}_{39} \mathrm{~N}_{3} \mathrm{O}_{3}$ & $\begin{array}{l}72.25 \\
(72.26)\end{array}$ & $\begin{array}{c}8.42 \\
(8.40)\end{array}$ & $\begin{array}{c}9.02 \\
(9.03)\end{array}$ & $\begin{array}{l}7.09-7.23(\mathrm{~m}, 10 \mathrm{H}, \mathrm{Ar}-\mathrm{H}) ; 1.43(\mathrm{~s}, 9 \mathrm{H}, \mathrm{Boc}) ; 7.90(\mathrm{~s}, 1 \mathrm{H}, \\
\mathrm{NH}-\mathrm{Boc}) ; 4.60(\mathrm{~s}, 1 \mathrm{H},-\mathrm{CH}-) ; 2.85\left(\mathrm{~s}, 4 \mathrm{H},-\mathrm{CH}_{2^{-}}\right) ; 2.49(\mathrm{~s}, 4 \mathrm{H} \text {, } \\
\left.-\mathrm{CH}_{2-}\right) ; 4.50(\mathrm{t}, 1 \mathrm{H},-\alpha \mathrm{CH}) ; 1.90\left(\mathrm{t}, 2 \mathrm{H}, \mathrm{CH}_{2^{-}}\right) ; 2.00(\mathrm{~m}, 1 \mathrm{H}, \\
-\gamma \mathrm{CH}-) ; 1.43\left(\mathrm{~d}, 6 \mathrm{H}, \delta \mathrm{CH}_{3}\right) .\end{array}$ \\
\hline $5 e$ & & 85 & $\mathrm{C}_{31} \mathrm{H}_{37} \mathrm{~N}_{3} \mathrm{O}_{3}$ & $\begin{array}{c}74.52 \\
(74.54)\end{array}$ & $\begin{array}{c}7.39 \\
(7.41)\end{array}$ & $\begin{array}{c}8.42 \\
(8.41)\end{array}$ & $\begin{array}{l}\text { 7.06-7.45 (m, } 15 \mathrm{H}, \mathrm{Ar}-\mathrm{H}) ; 1.43(\mathrm{~s}, 9 \mathrm{H}, \mathrm{Boc}) ; 7.98(\mathrm{~s}, 1 \mathrm{H}, \\
\mathrm{NH}-\mathrm{Boc}) ; 4.62(\mathrm{~s}, 1 \mathrm{H},-\mathrm{CH}-) ; 2.90\left(\mathrm{~s}, 4 \mathrm{H},-\mathrm{CH}_{2^{-}}\right) ; 2.62(\mathrm{~s}, 4 \mathrm{H}, \\
\left.-\mathrm{CH}_{2^{-}}\right) ; 4.52(\mathrm{t}, 1 \mathrm{H},-\alpha \mathrm{CH}-) ; 3.19\left(\mathrm{~d}, 2 \mathrm{H}, \beta \mathrm{CH}_{2^{-}}\right) .\end{array}$ \\
\hline $5 f$ & & 90 & $\mathrm{C}_{34} \mathrm{H}_{41} \mathrm{~N}_{3} \mathrm{O}_{5}$ & $\begin{array}{c}70.21 \\
(70.22)\end{array}$ & $\begin{array}{c}7.04 \\
(7.05)\end{array}$ & $\begin{array}{c}7.24 \\
(7.23)\end{array}$ & $\begin{array}{l}\text { 7.04-7.37 (m, } 15 \mathrm{H}, \mathrm{Ar}-\mathrm{H}) ; 1.43(\mathrm{~s}, 9 \mathrm{H}, \mathrm{Boc}) ; 7.80(\mathrm{~s}, 1 \mathrm{H}, \\
\mathrm{NH}-\mathrm{Boc}) ; 4.64(\mathrm{~s}, 1 \mathrm{H},-\mathrm{CH}-) ; 2.85\left(\mathrm{~s}, 4 \mathrm{H},-\mathrm{CH}_{2^{-}}\right) ; 2.54(\mathrm{~s}, 4 \mathrm{H}, \\
\left.-\mathrm{CH}_{2^{-}}\right) ; 4.41(\mathrm{~s}, 1 \mathrm{H},-\alpha \mathrm{CH}-) ; 1.89\left(\mathrm{q}, 2 \mathrm{H},-\beta_{\mathrm{CH}_{2}}\right) ; 2.16(\mathrm{t}, 2 \mathrm{H}, \\
\left.-\gamma \mathrm{CH}_{2^{-}}\right) ; 5.36\left(\mathrm{~s}, 2 \mathrm{H}, \mathrm{CH}_{2^{-}}\right) .\end{array}$ \\
\hline $5 \mathrm{~g}$ & $\begin{array}{c}\mathrm{C}^{-} \\
\mathrm{H}_{2} \\
\mathrm{H}\end{array}$ & 88 & $\mathrm{C}_{35} \mathrm{H}_{46} \mathrm{~N}_{4} \mathrm{O}_{3}$ & $\begin{array}{c}73.70 \\
(73.69)\end{array}$ & $\begin{array}{c}8.06 \\
(8.07)\end{array}$ & $\begin{array}{c}9.79 \\
(9.82)\end{array}$ & $\begin{array}{l}7.06-7.27(\mathrm{~m}, 15 \mathrm{H}, \mathrm{Ar}-\mathrm{H}) ; 1.43(\mathrm{~s}, 9 \mathrm{H}, \mathrm{Boc}) ; 7.96(\mathrm{~s}, 1 \mathrm{H}, \\
\mathrm{NH}-\mathrm{Boc}) ; 4.54(\mathrm{~s}, 1 \mathrm{H},-\mathrm{CH}-) ; 2.89\left(\mathrm{~s}, 4 \mathrm{H},-\mathrm{CH}_{2^{-}}\right) ; 2.50(\mathrm{~s}, 4 \mathrm{H}, \\
\left.-\mathrm{CH}_{2^{-}}\right) ; 4.71(\mathrm{t}, 1 \mathrm{H},-\alpha \mathrm{CH}) ; 1.48\left(\mathrm{q}, 2 \mathrm{H}, \beta_{\mathrm{CH}_{2}}\right) ; 1.55(\mathrm{q}, 2 \mathrm{H}, \\
\left.\gamma \mathrm{CH}_{2^{-}}\right) ; 1.42\left(\mathrm{q}, 2 \mathrm{H}, \delta \mathrm{CH}_{2^{-}}\right) ; 1.39\left(\mathrm{t}, 2 \mathrm{H}, \varepsilon \mathrm{CH}_{2^{-}}\right) ; 1.95(\mathrm{~s}, 1 \mathrm{H}, \\
\mathrm{NH}) ; 3.75\left(\mathrm{~d}, 2 \mathrm{H}, \mathrm{CH}_{2}\right) .\end{array}$ \\
\hline
\end{tabular}




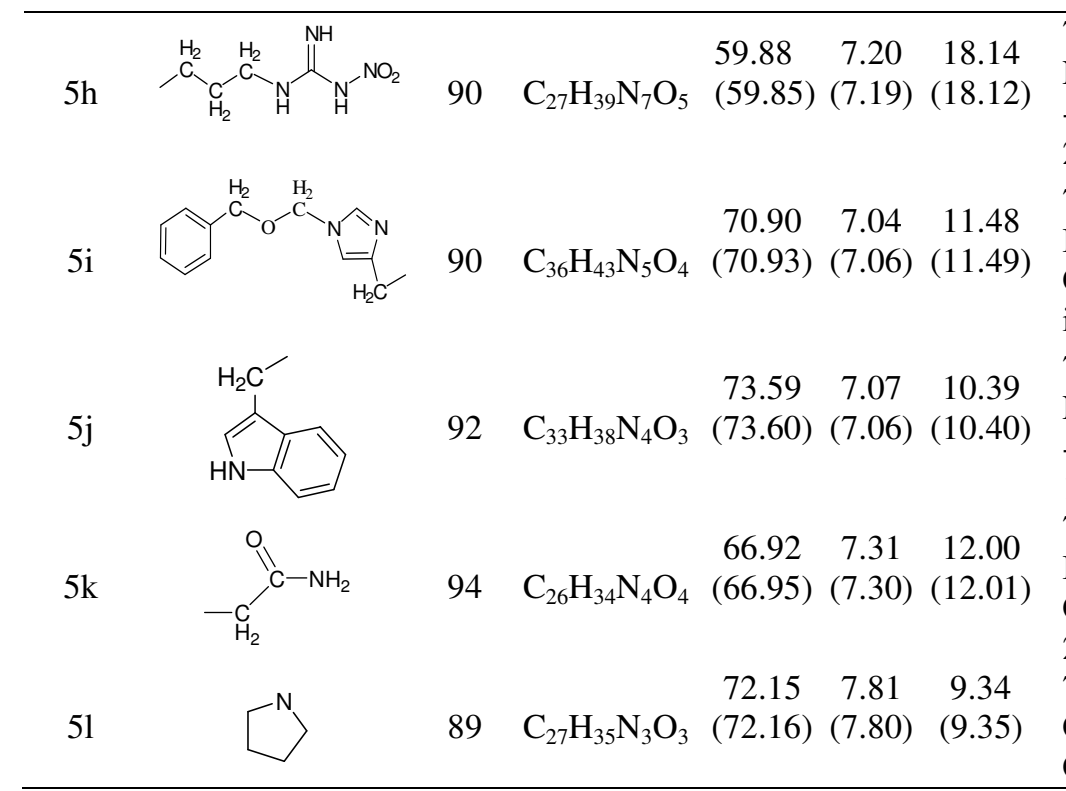

7.08-7.25 (m, 10H, Ar-H); 1.43 (s, 9H, Boc); 8.00 (s, 1H, NH-Boc); 4.73 (s, $1 \mathrm{H},-\mathrm{CH}-) ; 2.88$ (s, 4H, $\left.-\mathrm{CH}_{2}-\right) ; 2.59$ (s, $4 \mathrm{H}$, $\left.-\mathrm{CH}_{2}-\right) ; 4.54(\mathrm{t}, 1 \mathrm{H},-\alpha \mathrm{CH}-) ; 1.32\left(\mathrm{q}, 2 \mathrm{H}, \beta \mathrm{CH}_{2}-\right) ; 1.33$ (q, $\left.2 \mathrm{H}, \gamma \mathrm{CH}_{2}-\right) ; 2.59\left(\mathrm{t}, 2 \mathrm{H}, \delta \mathrm{CH}_{2}-\right) ; 2.0(\mathrm{~m}, 3 \mathrm{H}$, guanidine). 7.04-7.35(d, 15H, Ar-H); 1.43 (s, 9H, Boc); 8.00 (s, 1H, NHBoc); $4.54(\mathrm{~s}, 1 \mathrm{H},-\mathrm{CH}-) ; 2.90\left(\mathrm{~s}, 4 \mathrm{H},-\mathrm{CH}_{2}-\right) ; 2.50(\mathrm{~s}, 4 \mathrm{H},-$

$\mathrm{CH}_{2}$ ); 4.70 (s, $\left.1 \mathrm{H},-\alpha \mathrm{CH}-\right) ; 3.12\left(\mathrm{~s}, 2 \mathrm{H},-\beta \mathrm{CH}_{2}-\right) ; 6.55$ (s, $2 \mathrm{H}$, imidazole); $4.60\left(\mathrm{~s}, 2 \mathrm{H}, \mathrm{CH}_{2}\right) ; 5.61\left(\mathrm{~s}, 2 \mathrm{H}, \mathrm{CH}_{2}\right)$.

$7.04-7.30$ (m, 14H, Ar-H); 1.43 (s, 9H, Boc); 7.80 (s, 1H,

NH-Boc); 4.69 (s, 1H, -CH-); 2.92 (s, 4H, $\left.-\mathrm{CH}_{2}-\right) ; 2.48$ (s, 4H, $\left.-\mathrm{CH}_{2}-\right) ; 4.58(\mathrm{~s}, 1 \mathrm{H},-\alpha \mathrm{CH}-) ; 3.19\left(\mathrm{~d}, 2 \mathrm{H}, \beta \mathrm{CH}_{2}-\right) ; 10.12(\mathrm{~d}$, $1 \mathrm{H}, \mathrm{NH}$ of indole); 6.81 (d, 1H, - $\mathrm{CH}-)$. 7.04-7.19 (d, 10H, Ar-H); 1.45 (s, 9H, Boc); 7.98 (s, 1H, NHBoc); 4.67 (s, 1H, -CH-); 2.90 (s, 4H, - $\left.\mathrm{CH}_{2}-\right) ; 2.60$ (s, 4H, $\mathrm{CH}_{2}$ ); 4.48 (t, $\left.1 \mathrm{H},-\alpha \mathrm{CH}-\right) ; 2.81$ (t, $2 \mathrm{H},(\mathrm{CO}) \mathrm{CH}_{2}-$ ); 6.00 (s, $\left.2 \mathrm{H}, \mathrm{NH}_{2}\right)$.

7.05-7.22 (m, 10H, Ar-H); 1.43 (s, 9H, Boc); 4.65 (s, 1H, $\mathrm{CH}-) ; 2.88$ (s, 4H, - $\mathrm{CH}_{2}$ ); $2.55\left(\mathrm{~s}, 4 \mathrm{H},-\mathrm{CH}_{2^{-}}\right) ; 1.73(\mathrm{t}, 2 \mathrm{H},-$ $\left.\mathrm{CH}_{2}\right) ; 1.48$ (q, 2H, $\left.\mathrm{CH}_{2}\right) ; 3.1$ (t, 2H, - $\left.\mathrm{CH}_{2}\right) ; 3.9$ (t, $\left.1 \mathrm{H},-\mathrm{CH}-\right)$. 


\section{Deprotection of Boc group}

The Boc group was deblocked by treating the protected compounds $(1 \mathrm{mmol})$ with $4 \mathrm{~N} \mathrm{HCl}$ in dioxane $(10 \mathrm{~mL} / \mathrm{g}$ of the compound) for 1.5 hours. Excess $\mathrm{HCl}$ and dioxane were removed under reduced pressure, triturated with ether, filtered, washed with ether and dried (yield, 100\%).

\section{Antimicrobial assay}

\section{Antibacterial activity}

In vitro antibacterial assays were performed against Staphylococcus aureus, E coli, Klebesiella pnemoniae and Pseudomonas auregenosa by using agar well diffusion method ${ }^{25}$. All the synthesized compounds were tested in triplicate, streptomycin was used as positive control and water as negative control. The zone of inhibition area was measured in mm (Table 2).

\section{Antifungal activity}

In vitro antifungal assays were performed against Aspergillus niger, Aspergillus flavus and Fusarium monoliforme by using agar well diffusion method. ${ }^{26}$ The fungicidal activity of the synthesized compounds was assessed by comparing the zone of fungal growth in treated plates with that of control plates in mm (Table 2).

Table 2. Antibacterial and antifungal activity of the synthesized compounds against various bacterial and fungal strains.

\begin{tabular}{|c|c|c|c|c|c|c|c|}
\hline \multirow[b]{3}{*}{ Compounds $^{\mathrm{a}}$} & \multicolumn{7}{|c|}{ Inhibitory Zone (diameter) $\mathrm{mm}^{\mathrm{b}}$} \\
\hline & \multicolumn{4}{|c|}{ Bacterial Strains } & \multicolumn{3}{|c|}{ Fungal Strains } \\
\hline & $\begin{array}{c}\text { Staphylococcus } \\
\text { aureus }\end{array}$ & E.coli & $\begin{array}{l}\text { li Klebesiella } \\
\text { pnemoniae }\end{array}$ & $\begin{array}{c}\text { Pseudomonas } \\
\text { auregenosa }\end{array}$ & $\begin{array}{c}\text { Aspergillus } \\
\text { niger }\end{array}$ & $\begin{array}{l}\text { Aspergillus } \\
\text { flavus }\end{array}$ & $\begin{array}{c}\text { Fusarium } \\
\text { monoliforme }\end{array}$ \\
\hline 4 & 04 & 03 & 03 & 04 & 02 & 04 & 05 \\
\hline $5 \mathrm{a}$ & 08 & 08 & 05 & 06 & 05 & 04 & 05 \\
\hline $5 b$ & 07 & 08 & 06 & 07 & 06 & 05 & 04 \\
\hline $5 c$ & 09 & 08 & 07 & 08 & 02 & 07 & 05 \\
\hline $5 d$ & 09 & 08 & 09 & 10 & 06 & 06 & 05 \\
\hline $5 \mathrm{e}$ & 13 & 13 & 12 & 11 & 06 & 07 & 05 \\
\hline $5 f$ & 10 & 09 & 09 & 08 & 07 & 05 & 07 \\
\hline $5 g$ & 07 & 07 & 06 & 07 & 05 & 03 & 03 \\
\hline $5 \mathrm{~h}$ & 07 & 06 & 05 & 06 & 05 & 06 & 04 \\
\hline $5 \mathrm{i}$ & 10 & 09 & 07 & 09 & 07 & 08 & 07 \\
\hline $5 \mathrm{j}$ & 12 & 12 & 11 & 14 & 05 & 07 & 06 \\
\hline $5 \mathrm{k}$ & 05 & 04 & 05 & 06 & 04 & 05 & 06 \\
\hline 51 & 07 & 06 & 07 & 08 & 06 & 05 & 06 \\
\hline Streptomycin & 12 & 12 & 10 & 11 & -- & -- & -- \\
\hline Bavistin & -- & -- & -- & -- & 09 & 10 & 09 \\
\hline
\end{tabular}

\section{Results and Discussion}

We have synthesized new class of diphenylmethylpiperazine derivatives $\mathbf{5}(\mathbf{a}-\mathbf{l})$ by coupling of diphenylmethylpiperazine with $N$-protected Boc-amino acids using EDCI as a coupling agent, HOBt was used to avoid the racemization at the $C$-terminal amino acids, NMM was used to maintain the $\mathrm{pH}$ to 8 . The product obtained was gummy and characterized by TLC, elemental analysis and ${ }^{1} \mathrm{H}$ NMR. 
All the synthesized compounds 5(a-l) showed antibacterial activity against strains of both gram +ve and gram -ve bacteria such as Staphylococcus aureus, Klebesiella pnemoniae, Pseudomonas auregenosa and E coli and showed antifungal activity was against Aspergillus niger, Aspergillus flavus and Fusarium monoliforme. Among the synthesized compounds $\mathbf{5 e}$ and $\mathbf{5 j}$ showed equally good antibacterial activity as that of conventional antimicrobial drugs. Their enhancement of antibacterial activity may be due to the presence of aromatic and heterocyclic moiety of the amino acid residues. Even though amino acids and diphenylmethylpiperazine which taken in isolation were inactive or weakly active towards these bacterial strains, synthesized compounds showed activity.

\section{Conclusion}

In an effort to discover new diphenylmethylpiperazine analogues as antimicrobial molecule, we found that compounds $\mathbf{5 e}$ and $\mathbf{5 j}$ showed good antibacterial activity and all other compounds showed a moderate activity. But conversely some of the compounds $\mathbf{5 i}, \mathbf{5 e}, \mathbf{5 f}$ and $\mathbf{5 j}$ showed moderate antifungal activity and the remaining showed a mild antifungal activity. The results of the present study indicate that compounds $\mathbf{5 i}, \mathbf{5 j}, \mathbf{5 e}$ and $\mathbf{5 f}$ might be of interest for the identification of new antimicrobial molecules. Further work on conjugation of aromatic and heterocyclic moiety to the diphenylmethylpiperazine is in progress.

\section{Acknowledgement}

The authors wish to thank UGC New Delhi, CSIR New Delhi, Lady Tata Memorial Trust Mumbai and University of Mysore, Mysore for their financial assistance.

\section{References}

1. Gyoten M, Nagaya H, Fukuda S, Ashida Y and Kawano Y, Chem Pharm Bull Tokyo, 2003, 51(2), 122-133.

2. Magid A G, John A, Moyer Susan T N, Michael W and Usha P, J Med Chem., 1995, 38, 4026-4032.

3. Chaudhary P, Kumar R, Verma A K, Singh D, Bioorg Med Chem., 2006, 14, 1819-1826.

4. Hachiro S, Hiroo O, Yasuo A, Youichi I. and Yoshiharu Y, Japanese J Pharmacol., 2002, 89(1), 7-20.

5. Rebecca D P and Patricia M, Combinatorial Chemistry \& High Throughput Screenings, 2005, 8, 39-48.

6. Makoto K, Tomoko M, Koji Y, Masaki M, Nobuo K, Nobuyuki K, Kenichi K, Masato I, Yuji K, Katsuji O and Takayuki N, Bioorg \& Med Chem., 2003, 11, 3953-3963.

7. Makoto K, Tomoko M, Koji Y, Masaki M, Nobuo K, Nobuyuki K, Kenichi K, Masato I, Yuji K, Katsuji O and Takayuki N, Bioorg \& Med Chem., 2004, 12, 3069-3078.

8. He Zhao, Xiaoshu He, Andrew T, Diane H, Andrzej K, Robbin B, Renee P and Jan W F, Bioorg \& Med Chem Lett., 2002, 12, 3111-3115.

9. Brian D, Jessica P, Teresa P, Lee C, Brian M, Robin S, Julia H, Tracy B, Mary C, John S and Val G, Bioorg \& Med Chem Lett., 2003, 13, 3793-3796.

10. Rossen K, Steven A W, Sager J, Reamer R A, Askin D, Volante R P and Reider P J, Tetrahedron Lett., 1995, 36, 6419-6422.

11. David A, Kan K E, Kai R, Robert M P, Kenneth M W, Volante R P and Paul J R, Tetrahedron Lett., 1994, 35(5), 673-676.

12. Kawasaki N, Miyataka H, Nishiki M, Matsumato H, Inagaki N, Nagai H and Satoh T, Chem Pharma Bull., 1999, 47(2), 177-181.

13. Sugiyama N, Akahoshi S, Kuwahara M, Kajii, J Med Chem., 1994, 37, 1977-1982. 
14. Kumagai, US Patent, 1995, 5, 432, 179.

15. Kaneko, US Patent, 1994, 5, 304, 558.

16. Yung D K, Gilroy M L and Mahony D E, J Pharm Sci., 1978, 67(7), 900-905.

17. Yung D K, Mahony D E and Whitehouse L W, J Pharm Sci., 1971, 60(3), 386-389.

18. Suryanarayana V, Sandeep S, Menakshi J, Savitha S, Prati Pal S, Chaman Lal K and Rahul Jain, Bioorg Med Chem., 2004, 12, 239-247.

19. Sudhir R G, Rangan S R S, Krishna C A, J Med Chem., 1990, 33(5), 1505-1510.

20. Prasun K C, Philip L C, Michael J W, John A K, J Med Chem., 1983, 26, 633-638.

21. Ana C L L, Kezia P S, Ivone ADS, Janete M D A and Dalci J B, Eur J Med Chem., 2004, 39, 1059-1065.

22. Christian A, Emmanuelle V, Helene B, Claude P, Jean B, Bernard M, J Med Chem., 1984, 27, 1161-1166.

23. Hiroshi I, Maiko M, Boonma L and Tomoyo M, Int J Biol Macromol, 1995, 17(1), 21.

24. Song K S, Lee S H, Chun H J, Kim J Y, Jung M E, Ahn K, Kim S and lee J, Bioorg Med Chem., 2008, 16, 4035- 4051.

25. Perez C, Paul M and Bazerque P, Acta Biol Med Exp. 1990, 15, 113-115.

26. Singh I and Singh VP, Phytomorphology, 2000, 50(2), 151-157. 


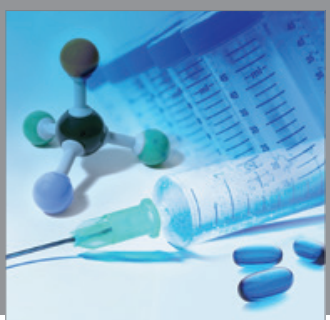

International Journal of

Medicinal Chemistry

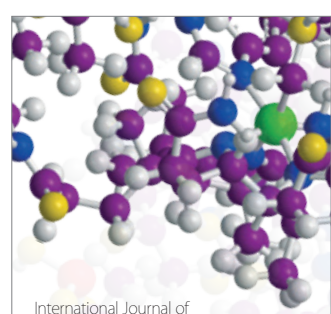

Carbohydrate Chemistry

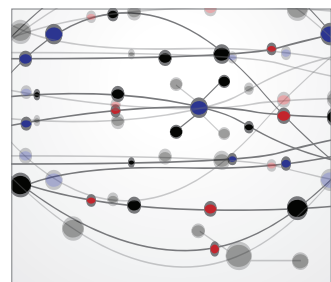

The Scientific World Journal
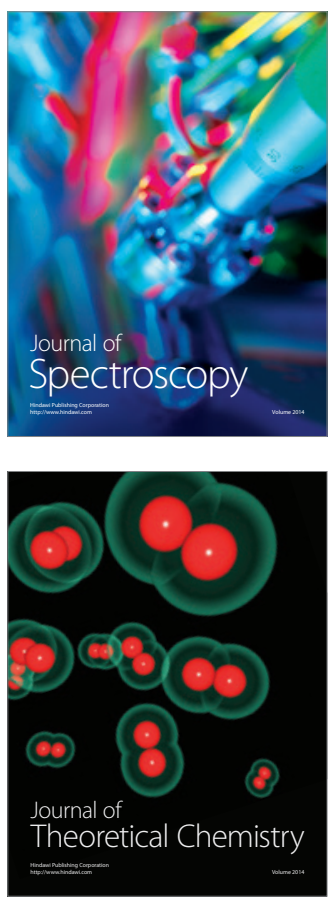
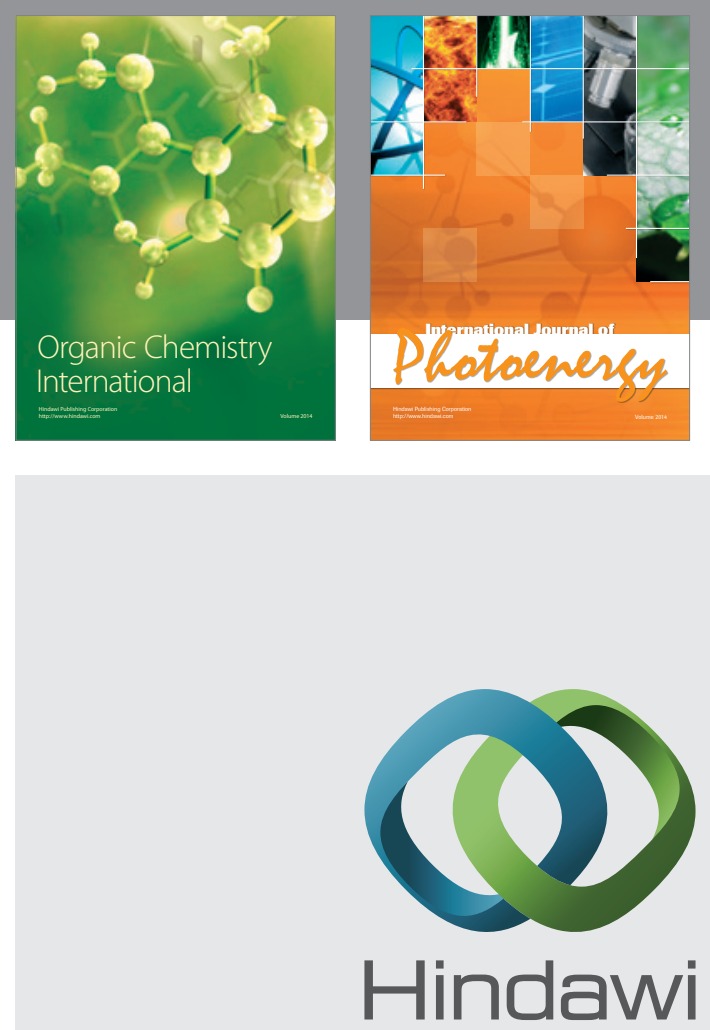

Submit your manuscripts at

http://www.hindawi.com
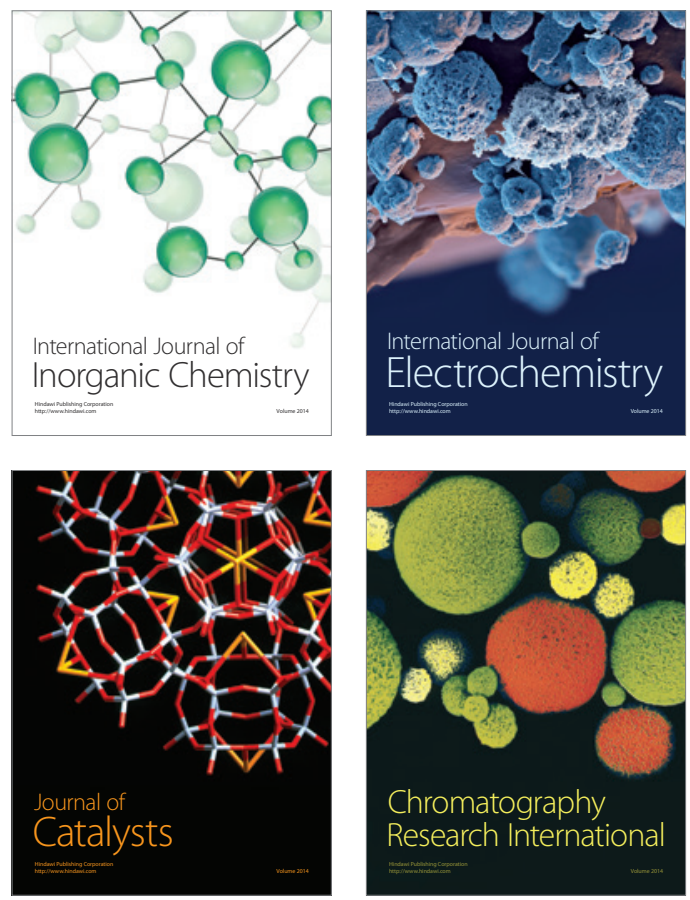
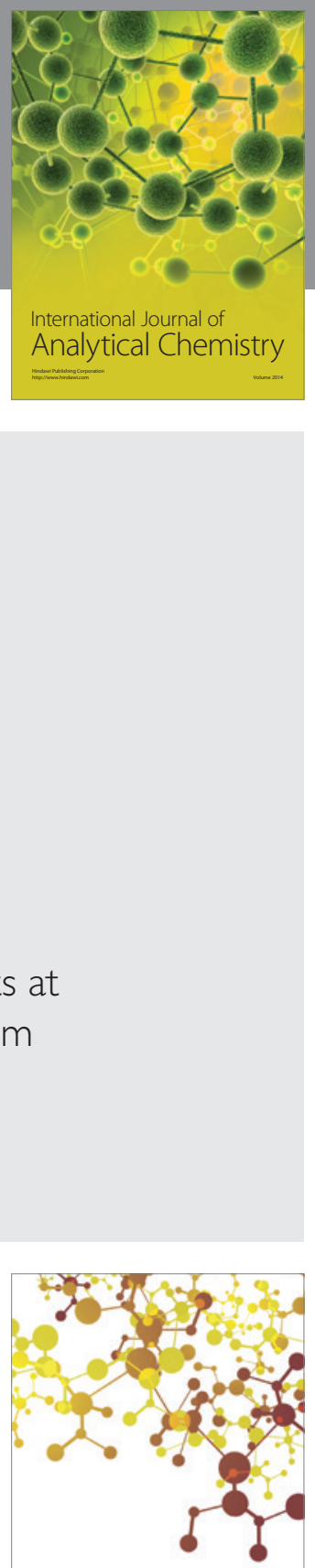

Journal of

Applied Chemistry
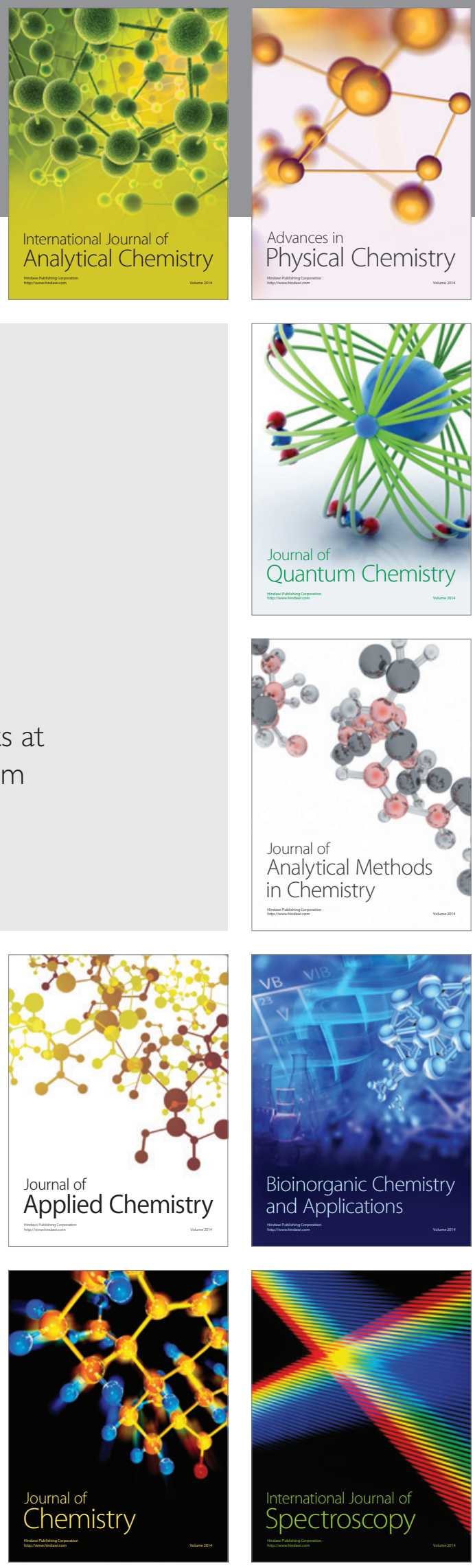